\title{
Modified RECIST (mRECIST) Assessment for Hepatocellular Carcinoma
}

\author{
Riccardo Lencioni, M.D., ${ }^{1}$ and Josep M. Llovet, M.D. ${ }^{2,3}$
}

The endpoint in cancer research is overall survival. Nonetheless, other potential surrogate endpoints, such as response rate and time to progression, are currently used. Measurement of response rate in hepatocellular carcinoma (HCC) has become a controversial issue. The World Health Organization (WHO) criteria underestimate the actual response rate; thus, they were amended in 2000 by a panel of experts convened by the European Association for the Study of the Liver (EASL) to take into account treatmentinduced tumor necrosis. Applying these guidelines, there was an association between response rate and outcome prediction. More recently, the Response Evaluation Criteria in Solid Tumors (RECIST) guideline was proposed as a method for measuring treatment response based on tumor shrinkage, which is a valuable measure of antitumor activity of cytotoxic drugs. This method was initially adopted by regulatory agencies, such as the U.S. Food and Drug Administration (FDA), for drug approval. However, anatomic tumor response metrics can be misleading when applied to molecular-targeted therapies or locoregional therapies in HCC. In 2008, a group of experts convened by the American Association for the Study of Liver Diseases (AASLD) developed a set of guidelines aimed at providing a common framework for the design of clinical trials in HCC and adapted the concept of viable tumor-tumoral tissue showing uptake in arterial phase of contrastenhanced radiologic imaging techniques - to formally amend RECIST. These amendments conformed the AASLD-JNCI (Journal of the National Cancer Institute) guidelines and are summarized and clarified in the current article. They are referred to herein as the modified RECIST assessment (mRECIST). Further studies are needed to confirm the accuracy of this measurement compared with conventional gold standards such as pathologic studies of explanted livers.

KEYWORDS: Hepatocellular carcinoma, RECIST, response assessment, time to progression magnetic resonance, computed tomography, AASLD-JNCI guidelines, imaging, targeted therapies

\footnotetext{
${ }^{1}$ Division of Diagnostic Imaging and Intervention, Department of Liver Transplantation, Hepatology, and Infectious Diseases, University of Pisa, Pisa, Italy; ${ }^{2}$ Barcelona Clinic Liver Cancer Group, Liver Unit, IDIBAPS, CIBERehd, Hospital Clínic, Barcelona, Spain; ${ }^{3}$ Mount Sinai Liver Cancer Program, Division of Liver Diseases, Mount Sinai School of Medicine, New York, New York.

Address for correspondence and reprint requests: Riccardo Lencioni, M.D., Division of Diagnostic Imaging and Intervention,

2, IT-56124 Pisa, Italy (e-mail: lencioni@med.unipi.it).

Hepatocellular Carcinoma; Guest Editors, Jordi Bruix, M.D., and Josep M. Llovet, M.D.

Semin Liver Dis 2010;30:52-60. Copyright (C) 2010 by Thieme Medical Publishers, Inc., 333 Seventh Avenue, New York, NY 10001, USA. Tel: +1 (212) 584-4662.

DOI: http://dx.doi.org/10.1055/s-0030-1247132.

ISSN 0272-8087. 


\section{BACKGROUND ON RESPONSE ASSESSMENT AND NEED OF GUIDELINES IN HCC RESEARCH}

The endpoint in cancer research is overall survival. Nonetheless, tumor response and time to progression have been considered pivotal for surrogate assessment of efficacy. Tumor response was initially measured according to the World Health Organization (WHO) criteria, ${ }^{1}$ and afterwards according to the Response Evaluation Criteria in Solid Tumors (RECIST) guideline. ${ }^{2} \mathrm{WHO}$ and RECIST define standard measurement methods for converting radiology image observations into a quantitative and statistically tractable framework for measuring the response of tumor size to therapy. Both methods offer simple approaches to determining anatomic size and lesion changes during treatment as an indicator of response. Target lesions are measured using either the bilinear product approach (WHO) or single linear summation (RECIST).

The WHO criteria and RECIST were designed primarily for the evaluation of cytotoxic agents. They do not address measures of antitumor activity other than tumor shrinkage. As acknowledged in the original RECIST publication, assessments based solely on changes in tumor size can be misleading when applied to other anticancer drugs, such as molecular-targeted therapies, or other therapeutic interventions. ${ }^{2}$ In the case of hepatocellular carcinoma (HCC), recent studies have shown a poor correlation between the clinical benefit provided by new agents such as sorafenib or by locoregional interventional therapies and conventional methods of response assessment. ${ }^{3,4}$

In 2000, a panel of experts on HCC convened by the European Association for the Study of the Liver (EASL) amended the response criteria to take into account tumor necrosis induced by treatment. ${ }^{5}$ That panel considered estimation of the reduction in viable tumor area using contrast-enhanced radiologic imaging to be the optimal method to assess treatment response. Viable tumor was defined as uptake of contrast agent in the arterial phase of dynamic computed tomography (CT) or magnetic resonance imaging (MRI). The concept of viable tumor proposed by the EASL panel has been subsequently endorsed by the American Association for the Study of Liver Diseases (AASLD). The AASLD practice guideline on the management of HCC issued in 2005 stated that the evaluation of the treatment response should take into account the induction of intratumoral necrotic areas in estimating the decrease in tumor load, and not just a reduction in overall tumor size. $^{6}$

Due to the growing complexity of trial design and assessment of benefits in the HCC arena, a group of experts convened by the AASLD developed a set of guidelines aimed at providing a common framework for the design of clinical trials. ${ }^{7}$ These AASLD-JNCI
(Journal of the National Cancer Institute) guidelines included for the first time a formal modification of the assessment of response based on the RECIST criteria, and aimed to translate the concept of viable tumor posed by the previous guidelines ${ }^{5,6}$ in a more updated framework. ${ }^{7}$ These amendments are referred to in the current article as modified RECIST assessment (mRECIST) for HCC. First, we will summarize the conclusions of the panel in terms of trial design in HCC; second, we will address the issue of standardizing imaging techniques for response assessment; finally, we will describe in detail the proposed amendments.

\section{DESIGN OF CLINICAL TRIALS IN HEPATOCELLULAR CARCINOMA}

The increasing amount of clinical trials ongoing in HCC has raised the need to have a common frame to test novel drugs accepted by all disciplines. As a consequence, new guidelines on the design of clinical trial and endpoints in $\mathrm{HCC}$ have been reported by a multidisciplinary panel of experts including hepatologists, surgeons, oncologists, radiologists, trialists, and experts in quality of life and regulatory issues. ${ }^{7}$ These statements will evolve as new evidence becomes available, including more precise information on natural history of HCC, new drugs or predictive biomarkers. The critical key points are summarized in Table 1.

1. Endpoints. Survival and time to recurrence were proposed as primary endpoints for phase III studies assessing primary and adjuvant therapies, respectively. Composite endpoints such as disease-free survival (DFS) or progression-free survival (PFS) are vulnerable in $\mathrm{HCC}$ research, particularly when the target population is ill-defined, and should be included as secondary endpoints. Quality of life assessment in HCC research suffers from the lack of a reliable, standardized, and adequately validated questionnaire, and thus it is currently recommended as ancillary information.

2. Importance of phase II studies. Randomized phase II studies were considered pivotal prior to conducting phase III trials in HCC. These studies classically consider response rate as the gold standard for efficacy. However, the advent of molecular compounds has changed the paradigm of trial design because there is no direct correlation between response and outcome prediction (See Llovet et $\mathrm{al}^{7}$ for details). Consequently, response rate was formally discouraged as a reliable endpoint to capture benefit in phase II studies. Time to progression (TTP) was endorsed as the most reliable time to event endpoint in phase II studies. Finally, phase I studies should specifically include HCC patients with Child-Pugh A cirrhosis to estimate the exact dose, toxicity, and liver-related 
Table 1 Summary of Conclusions of the AASLD-JNCI Guidelines for Trial Design in HCC ${ }^{7}$

Endpoints: Survival or time to recurrence (phase III), time to progression (phase II)

Trial strategy: Test drugs in the setting of randomized phase II before moving to phase III

HCC Classification: BCLC staging system is recommended for selection of target population and stratification

Assessment of response and TTP: Should follow the AASLD-JNCI amendments, which are summarized in the current article

\author{
Standard of care (control arm) and drugs tested: \\ HCC Subclass (Standard of Care) \\ BCLC 0 or A-Early stages (resection, transplantation, \\ local ablation) \\ BCLC B-Intermediate stage (Chemoembolization-TACE) \\ BCLC C-Advance stage (Sorafenib)
}

\begin{tabular}{ll}
\multicolumn{2}{c}{ Testing Novel Drugs } \\
1st Line Treatment & 2nd Line Treatment* \\
Adjuvant: drug vs. placebo & - \\
& - \\
TACE vs. TACE + drug & \\
TACE vs. drug or device & \\
$\begin{array}{l}\text { Sorafenib vs. sorafenib }+ \text { drug } \\
\text { Sorafenib vs. drug }\end{array}$ & Drug vs. placebo
\end{tabular}

* In case of failure to standard of care.

† Head to head comparisons with standard of care are only justified if phase II data are very promising.

BCLC, Barcelona-Clinic Liver Cancer staging system; TACE, transarterial chemoembolization. event risk not captured by phase I studies including patients with a variety of neoplasms. This will minimize the nondesirable events as a result of the natural history of cirrhosis that might lead to death due to bleeding, hepatorenal syndrome, infections, and other complications. ${ }^{8}$ This population selection will ensure that more than $90 \%$ of deaths in the first 2 years will be due to tumor progression. ${ }^{8}$ This is of relevance when using nonrecommended composite endpoints, such as PFS, as the contamination of the endpoint with deaths unrelated to the effect of the drug should be tightly controlled.

3. HCC Classification for trial stratification. The panel endorsed the BCLC staging classification for the selection of the target population and/or for the stratification of patients prior randomization. ${ }^{7,9} \mathrm{Con}-$ sequently, the selection of unresectable HCC patients is discouraged for entering patients in phase II studies. By using a common classification, it will be possible to easily interpret the value of outcome data generated by different investigators.

4. Assessment of tumor response and time to progression should follow the amendments of RECIST endorsed by the AASLD-JNCI panel. ${ }^{7}$

5. Standard of care (control arm) and drugs tested. The control arm for clinical trials should be the standard of care: chemoembolization for intermediate HCCs and sorafenib for advanced cases.,10 Therefore, for the assessment of first-line systemic treatments for advanced HCC a design adding a new agent to sorafenib versus sorafenib alone is recommended. Comparison of single agents head to head with the standard of care therapy might jeopardize the recruitment of patients due to ethical reasons, unless the novel agent showed very promising efficacy in early phase II studies. For second-line treatments, the new agent should be randomized against placebo/best supportive care, and the selection criteria should include patients with contraindications or failures to sorafenib. Randomized studies testing molecular-targeted therapies should optimally include biomarker analysis (tissue and/or serum samples) to enable the identification of molecular markers of response and for pharmacokinetic purposes, as reported in other cancers.

\section{SUMMARY OF THE MRECIST ASSESSMENT OF RESPONSE AND PROGRESSION}

We are expanding and detailing herein some of the main recommendations posed by the AASLD-JNCI guidelines position paper. ${ }^{7}$

\section{Standardizing Response Assessment}

\section{IMAGE ACQUISITION}

Optimization of image acquisition protocols and consistency in the use of the same protocol throughout follow-up examinations are key for proper application of mRECIST. Patients can be followed with either contrast-enhanced spiral computed tomography (CT) preferably with use of multislice scanners-or contrastenhanced dynamic magnetic resonance imaging (MRI). The administration of intravenous contrast is recommended for all CT or MRI studies if not medically contraindicated. In contrast-enhanced studies, it is mandatory to obtain a dual-phase imaging of the liver. Every effort should be made to time the contrast administration so that high-quality arterial-phase imaging is obtained throughout the liver on the first run, and 
high-quality portal venous-phase imaging is obtained throughout the liver on the second run. Delayed imaging obtained in the equilibrium phase may be useful, but it is not mandatory and should be done only if it is part of clinical practice. For multidetector CT scanners that are capable of acquiring very thin slices, it is necessary to keep in mind that it is mandatory to use contiguous slices for image read and interpretation, to avoid missing small lesions. For example, the analysis of contiguous slices with traditional $5 \mathrm{~mm}$ thickness and $5 \mathrm{~mm}$ reconstruction interval is acceptable; however, the analysis of $2.5 \mathrm{~mm}$ thickness slices at $5 \mathrm{~mm}$ intervals is not acceptable.

\section{IMAGE INTERPRETATION}

To properly use the proposed mRECIST for HCC to assess response rates and time to progression in HCC clinical trials and to ensure comparability across studies, uniform image acquisition parameters, rigorous quality control, and independent blinded multireader assessments are mandatory. Therefore, the expert panel recommended adopting a centralized radiologic review for image interpretation rather than base the assessment on the image evaluation performed by local investigators. Independent radiologists will be responsible for performing qualitative and quantitative assessments of imaging data. They will assess baseline imaging to determine the overall tumor burden and use this as a comparator for subsequent measurements. Tumor response will then be determined for each follow-up imaging time point. Overall response assessment includes, according to RECIST, evaluation of target lesions response, nontarget lesions response, and new lesions.

\section{ASSESSMENT OF TUMOR LESIONS AT BASELINE}

According to RECIST, tumor lesions are categorized at baseline as follows: measurable (lesions that can be accurately measured in at least one dimension as $\geq 1 \mathrm{~cm}$ with a spiral CT scan) or nonmeasurable [all other lesions, including small lesions (longest diameter $<1 \mathrm{~cm}$ with spiral CT scan) and truly nonmeasurable lesions]. The original RECIST publication states that all measurable lesions up to a maximum of five lesions per organ and 10 lesions in total, representative of all involved organs, should be identified as target lesions and recorded and measured at baseline. The recent 1.1 release of RECIST has reduced the number of lesions to select as target lesions to a maximum of two lesions per organ and five lesions in total. ${ }^{11}$ In fact, analyses on a large prospective database has shown that assessment of five versus 10 lesions per patient did not affect the overall response rate, and that progression-free survival was only minimally affected. ${ }^{12}$ Target lesions should be selected on the basis of their size (those with the longest diameter) and their suitability for accurate repeated measurements. All other lesions (or sites of disease) should be identified as nontarget lesions and should also be recorded at baseline. Measurements of these lesions are not required, but the presence or absence of each should be noted throughout follow-up.

It is our understanding that the measurement of the longest viable tumor diameter for the assessment of response according to mRECIST can be only applied in case of typical lesions. Conversely, for non- enhancing atypical lesions, as well as for any extrahepatic neoplastic niches, the measurements of the longest overall tumor diameter as per conventional RECIST should prevail.

To be selected as a target lesion using mRECIST, an HCC lesion should meet all the following criteria:

- The lesion can be classified as a RECIST measurable lesion (i.e., the lesion can be accurately measured in at least one dimension as $1 \mathrm{~cm}$ or more).

- The lesion is suitable for repeat measurement.

- The lesion shows intratumoral arterial enhancement on contrast-enhanced CT or MRI.

It is important to point out that only well-delineated, arterially enhancing lesions can be selected as target lesions for mRECIST. This may not be the case of infiltrative-type HCC. Infiltrative-type HCC should be considered as a nontarget lesion when the mass shows ill-defined borders and therefore does not appear to be suitable for accurate and repeat measurements. HCC lesions previously treated with locoregional or systemic treatments may or may not be considered as suitable to be selected as target lesions for mRECIST: if the lesion shows a well-delineated area of viable (contrast enhancement in the arterial phase) tumor that is at least $1 \mathrm{~cm}$ in longest diameter, then it can be selected as a target lesion. In contrast, if the lesion is poorly demarcated or exhibits atypical enhancement as a result of the previous intervention, then it cannot be selected as a target lesion for mRECIST.

\section{Defining Treatment Response and Tumor Progression}

\section{TARGET LESIONS RESPONSE}

According to RECIST, complete response is the disappearance of all target lesions; partial response is at least a $30 \%$ decrease in the sum of the longest diameter of target lesions, taking as reference the baseline sum longest diameter; progressive disease is at least a $20 \%$ increase in the sum of the longest diameter of target lesions, taking as reference the smallest sum longest diameter recorded since when treatment started or the appearance of one or more new lesions; stable disease is neither sufficient shrinkage to qualify for partial response 
Table 2 Assessment of Target Lesion Response: Conventional RECIST and mRECIST Assessment for HCC Following the AASLD-JNCI Guideline

\begin{tabular}{|c|c|}
\hline RECIST & mRECIST for HCC \\
\hline $\mathrm{CR}=$ Disappearance of all target lesions & $\begin{array}{l}\mathrm{CR}=\text { Disappearance of any intratumoral arterial enhancement } \\
\text { in all target lesions }\end{array}$ \\
\hline $\begin{array}{l}\text { PR }=\text { At least a } 30 \% \text { decrease in the sum of diameters } \\
\text { of target lesions, taking as reference the baseline } \\
\text { sum of the diameters of target lesions }\end{array}$ & $\begin{array}{l}\mathrm{PR}=\text { At least a } 30 \% \text { decrease in the sum of diameters of viable } \\
\text { (enhancement in the arterial phase) target lesions, taking as } \\
\text { reference the baseline sum of the diameters of target lesions }\end{array}$ \\
\hline $\begin{array}{l}\mathrm{SD}=\text { Any cases that do not qualify for either partial } \\
\text { response or progressive disease }\end{array}$ & $\begin{array}{l}\mathrm{SD}=\text { Any cases that do not qualify for either partial response } \\
\text { or progressive disease }\end{array}$ \\
\hline $\begin{array}{l}\mathrm{PD}=\mathrm{An} \text { increase of at least } 20 \% \text { in the sum of the } \\
\text { diameters of target lesions, taking as reference the } \\
\text { smallest sum of the diameters of target lesions } \\
\text { recorded since treatment started }\end{array}$ & $\begin{array}{l}\mathrm{PD}=\text { An increase of at least } 20 \% \text { in the sum of the diameters } \\
\text { of viable (enhancing) target lesions, taking as reference the } \\
\text { smallest sum of the diameters of viable (enhancing) target } \\
\text { lesions recorded since treatment started }\end{array}$ \\
\hline
\end{tabular}

AASLD, American Association for the Study of Liver Diseases; JNCI, Journal of the National Cancer Institute; HCC, hepatocellular carcinoma; mRECIST, modified Response Evaluation Criteria in Solid Tumors; CR, complete response; PR, partial response; SD, stable disease; PD, progressive disease.

nor sufficient increase to qualify for progressive disease, taking as reference the smallest sum longest diameter since the treatment started.

The mRECIST for HCC has introduced the following amendments to RECIST in the determination of tumor response for target lesions (Table 2):

- Complete response: the disappearance of any intratumoral arterial enhancement in all target lesions

- Partial response: at least a 30\% decrease in the sum of diameters of viable (contrast enhancement in the arterial phase) target lesions, taking as reference the baseline sum of the diameters of target lesions

- Progressive disease: an increase of at least 20\% in the sum of the diameters of viable (enhancing) target lesions, taking as reference the smallest sum of the diameters of viable (enhancing) target lesions recorded since the treatment started

- Stable disease: any cases that do not qualify for either partial response or progressive disease
The measurement of the longest diameter of the viable tumor may be challenging in lesions showing partial internal necrosis (Fig. 1). The following points should be taken into account in such cases:

- The measurement of the viable tumor should be performed on CT or MRI obtained in the arterial phase, when the contrast between viable vascularized tumor tissue and nonenhancing necrotic tissue is the highest.

- The longest diameter of the viable tumor is not necessarily located in the same scan plane in which the baseline diameter was measured: a thorough careful evaluation of the CT or MRI scans is required.

- The measurement of the viable tumor diameter should not include any major intervening areas of necrosis.

It is important to point out that a reduction of at least $30 \%$ in the diameter of the viable tumor (the threshold required to declare partial response according
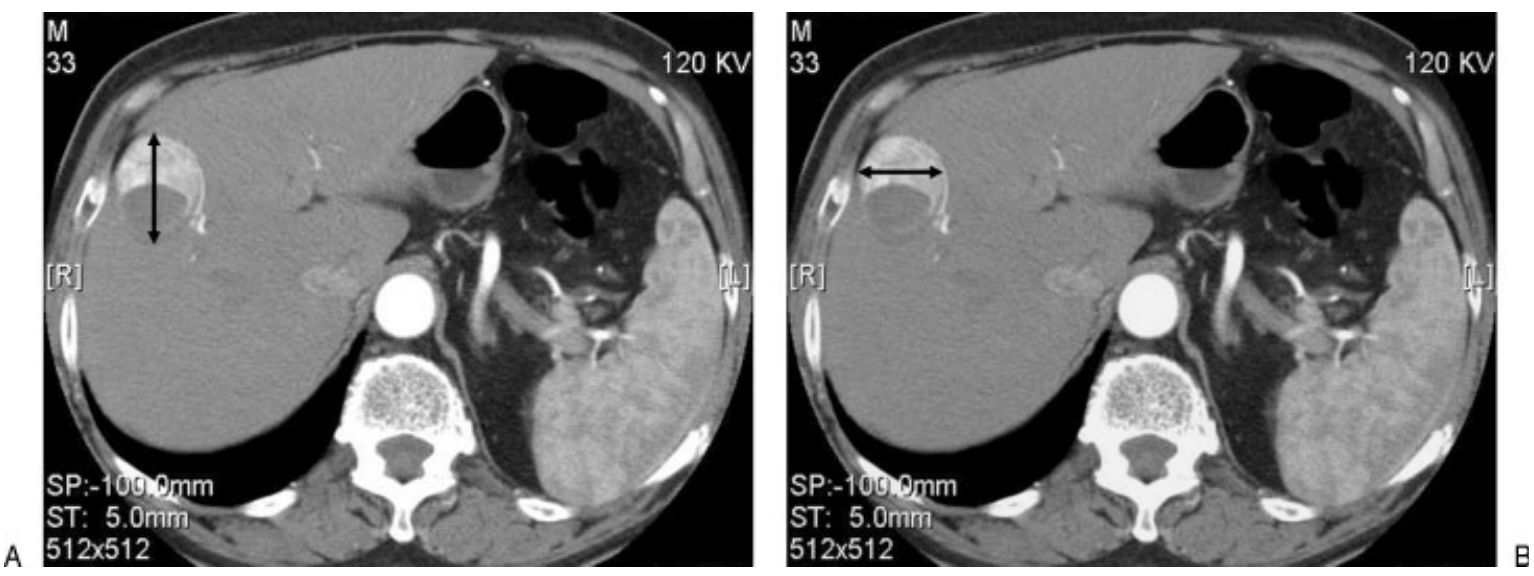

Figure 1 Application of mRECIST assessment for hepatocellular carcinoma (HCC). Target tumor response measurements on arterial-phase computed tomography (CT) scans. (A) Measurement of longest overall tumor diameter according to conventional RECIST, and (B) measurement of longest viable tumor diameter according to mRECIST for HCC. 
to mRECIST) corresponds to a decrease of $65 \%$ in viable tumor volume. In contrast, an increase of at least $20 \%$ in the diameter of the viable tumor (the threshold required to declare progressive disease according to mRECIST) corresponds to an increase of at least 73\% in viable tumor volume. The panel acknowledged that direct volumetric measurement to identify partial response and progression should be a priority in future clinical trial research.

\section{NONTARGET LESIONS RESPONSE}

The RECIST guideline provides the following definitions of the criteria used to determine the objective tumor response for nontarget lesions: complete response is the disappearance of all nontarget lesions; incomplete response/stable disease is the persistence of one or more nontarget lesions; and progressive disease is the appearance of one or more new lesions and/or unequivocal progression of existing nontarget lesions.

According to mRECIST for HCC, tumor necrosis should be taken into account when assessing the response of nontarget lesions. The disappearance of intratumoral arterial enhancement in nontarget lesions should be considered equivalent to the disappearance of nontarget lesions, and therefore, should declare complete response of nontarget lesions. The persistence of intratumoral arterial enhancement in one or more nontarget lesions should be considered equivalent to persistence of one or more nontarget lesions, and therefore, should declare incomplete response / stable disease. The appearance of one or more new lesions and/or unequivocal progression of existing nontarget lesions should declare progressive disease.

Special recommendations for the assessment of tumor response in nontarget lesions in patients with $\mathrm{HCC}$ and cirrhosis can be made regarding the following points:

1. Portal vein thrombosis. Malignant portal vein thrombosis should be considered a nonmeasurable lesion due to the difficulty in performing consistent measurements of the malignant thrombus during the course of the treatment. Measurements of the extent of the malignant thrombus may be impaired by the possible presence of a bland component of the thrombosis.

2. Porta hepatis lymph node. Lymph nodes detected at the portal hepatis can be considered as malignant if the lymph node short axis is at least $20 \mathrm{~mm}$. Evidence of reactive lymph nodes at the porta hepatis, in fact, is a common finding in patients with cirrhosis regardless of the presence of an HCC. The short axis of the node is the diameter normally used by radiologists to judge if a node is involved by solid tumor.

3. Pleural effusion and ascites. The original RECIST publication specifies that cytologic confirmation of the neoplastic nature of any effusion that appears or worsens during treatment is required when the measurable tumor has met criteria for response or stable disease. Under such circumstances, the cytologic examination of the fluid collected will permit differentiation between response or stable disease (an effusion may be a side effect of the treatment) and progressive disease (if the neoplastic origin of the fluid is confirmed). The mRECIST for HCC panel of experts considered this issue to be of high importance in the setting of $\mathrm{HCC}$ in cirrhosis. The emergence or the increase in ascites is a common event during the course of treatment in a cirrhotic patient, which may be due to worsening of the underlying chronic liver disease and be unrelated to cancer progression. ${ }^{8}$ Other effusions, such as pleural effusion, may also be unrelated to cancer progression and be caused by the liver insufficiency. Thus, the mRECIST for HCC emphasizes that cytopathologic confirmation of the neoplastic nature of any effusion (particularly ascites) that appears or worsens during treatment is required when the measurable tumor has met criteria for response or stable disease. It has to be underlined that peritoneal carcinomatosis is a very rare event in HCC.

\section{NEW LESIONS}

Characterization of a newly detected focal liver lesion as true $\mathrm{HCC}$ is a challenging issue in the setting of cirrhosis because pathologic abnormalities related to cirrhosis changes - such as large regenerative nodules and dysplastic nodules-may be indistinguishable from a small tumor. Moreover, the clear-cut separation of the hepatic phases of liver enhancement routinely achieved by state-of-the-art CT or MRI creates additional problems in a cirrhotic liver, mostly related to the presence of perfusion abnormalities resulting in areas of abnormal liver enhancement. In most cases, such perfusion abnormalities are detected as arterially hyperenhancing areas caused by a selective impairment of the portal venous feeding. Such perfusion abnormalities may ultimately mimic or conceal focal liver lesions; hence, they represent an additional major source for interpretation errors.

The AASLD practice guideline for the clinical management of $\mathrm{HCC}$ has recommended strict criteria for the imaging diagnosis of HCC in cirrhosis. ${ }^{6}$ Noninvasive diagnostic criteria of $\mathrm{HCC}$ can be made without histology in lesions of at least $1 \mathrm{~cm}$ in diameter showing characteristic vascular features of $\mathrm{HCC}$ - arterial hypervascularization with washout in the portal venous or the late phase - at dynamic imaging studies. For diagnostic purposes, two imaging techniques-CT and MRI-are required for such a confirmation in tumors of 1 to $2 \mathrm{~cm}$ in diameter, and one imaging technique in tumors beyond $2 \mathrm{~cm}$ in cirrhotic patients. 
A
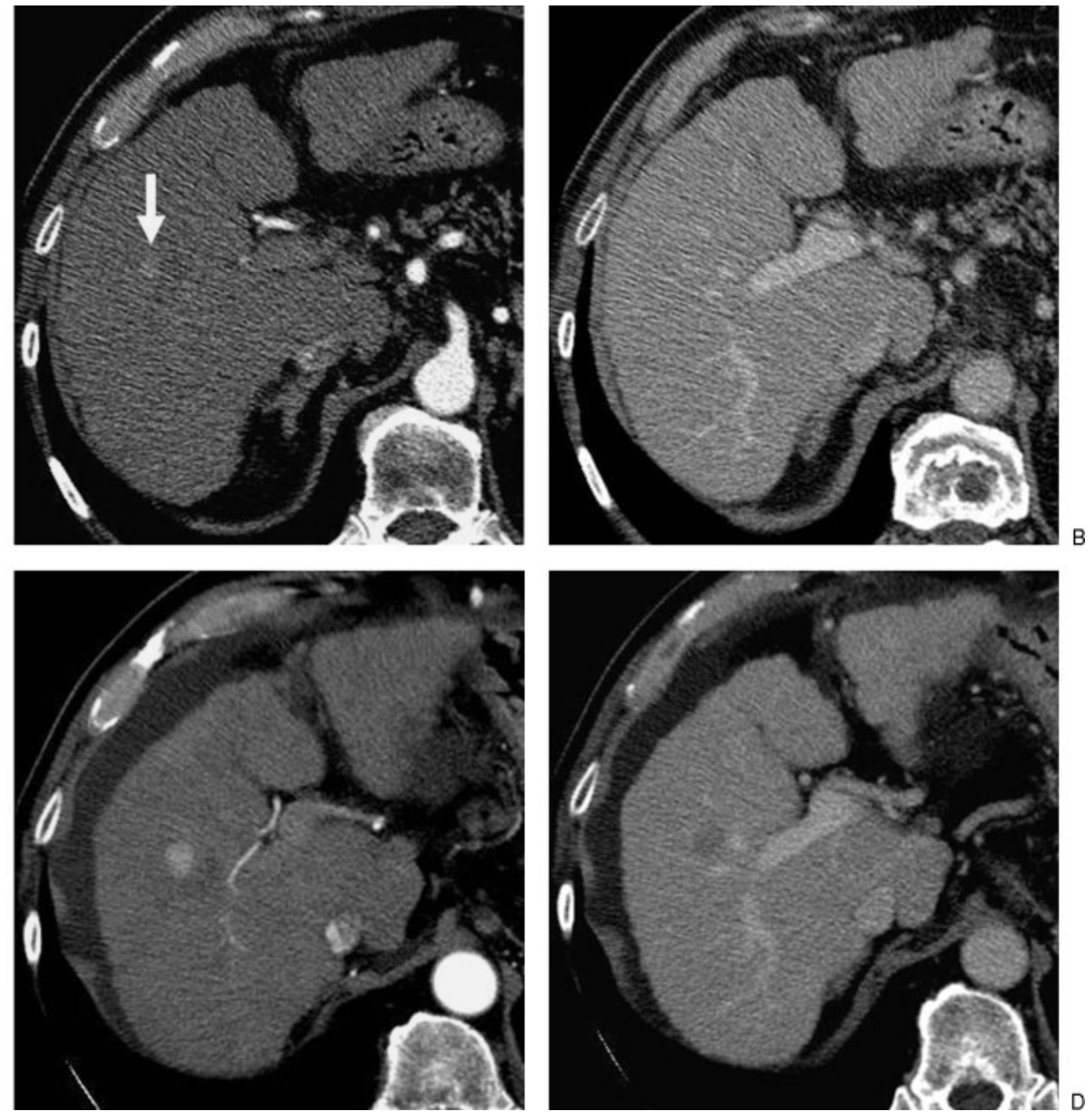

Figure 2 Application of mRECIST assessment for hepatocellular carcinoma (HCC). New lesion. Computed tomography (CT) scans obtained in an HCC patient's follow-up after treatment (main tumor not shown). On scans obtained at time point 1 ( $A$, arterial phase; $B$, venous phase), a new lesion is identified (arrow). The tiny lesion is smaller than $1 \mathrm{~cm}$; therefore, it must be considered equivocal. On CT scans obtained at time point 2 (C, arterial phase; D, venous phase), the tumor has become larger than $1 \mathrm{~cm}$ and shows the characteristic vascular pattern of HCC (arterial hypervascularization with venous washout). Although the criteria for diagnosing the lesion as HCC were fulfilled only at time point 2, progression must be declared in retrospect at time point 1 , that is at the time the lesion was first detected.

In the assessment of tumor progression, these concepts have been adopted by the mRECIST assessment proposal, considering some specificities for the frame of progression mode (Fig. 2):

- A newly detected hepatic nodule will be classified as HCC - and therefore will be declared as evidence of progression-when its longest diameter is at least $1 \mathrm{~cm}$ and the nodule shows the typical vascular pattern of HCC on dynamic imaging, that is, hypervascularization in the arterial phase with washout in the portal venous or late venous phase.

- Liver lesions larger than $1 \mathrm{~cm}$ that do not show a typical vascular pattern can be diagnosed as HCC by evidence of at least 1-cm-interval growth in subsequent scans.

- An individual radiologic event will be adjudicated in retrospect as progression at the time it was first detected by imaging techniques, even if strict criteria were fulfilled only on subsequent radiologic testing.

\section{Overall Response Assessment}

In mRECIST for HCC, identical to conventional RECIST, overall patient response is a result of the combined assessment of target lesions, nontarget lesions, and new lesions (Table 3). It is important to point out that appearance of one or more new lesions declares 
Table 3 Overall Response Assessment in mRECIST: Responses for All Possible Combinations of Tumor Responses in Target and Nontarget Lesions with or without the Appearance of New Lesions

\begin{tabular}{llll}
\hline Target Lesions & Nontarget Lesions & New Lesions & Overall Response \\
\hline CR & CR & No & CR \\
CR & IR/SD & No & PR \\
PR & Non-PD & No & PR \\
SD & Non-PD & No & PD \\
PD & Any & Yes or no & PD \\
Any & PD & Yes or no & PD \\
Any & Any & Yes & \\
\hline
\end{tabular}

mRECIST, modified Response Evaluation Criteria in Solid Tumors; CR, complete response; PR, partial response; IR, incomplete response; SD, stable disease; PD, progressive disease.

progression whatever the response of target and nontarget lesions. Overcalling of equivocal lesions as new HCC, therefore, has a major impact on the outcome of studies with a radiologic endpoint, such as tumor response or time to progression. Hence, any newly detected focal liver lesion that does not meet the criteria reported above should be considered equivocal and not conclusive for disease progression.

\section{FUTURE RESEARCH AND VALIDATION}

The adequate assessment of response and time to progression in the $\mathrm{HCC}$ arena is an evolving matter. In the early nineties, the $\mathrm{HCC}$ community was not following the conventional WHO criteria for assessment of response due to the alarming underestimation of responses, particularly following local ablation or chemoembolization treatments. This scenario changed with the introduction of the concept of viable tumor, which could be measured by imaging techniques as the area of uptake in the arterial phase of contrast-enhanced radiologic explorations. Correlation of these measurements with explanted liver lesions confirmed the validity of the concept. ${ }^{13}$ The advent of the conventional RECIST criteria in 2000 and the consequent adoption of these rules by regulatory agencies such as the FDA changed the landscape of tumor response assessment in HCC. Again, the classical RECIST only considered tumor shrinkage in a single diameter as the sole measure of response, hampering the proper assessment of response in complex tumors, such as liver cancer. Other limitations of classical RECIST, such as the definition of progression based on new lesions and presence of ascites, certainly could not be adopted by the scientific community. Nonetheless, in the setting of the first positive trial in $\mathrm{HCC}$ research with novel molecular targeted therapies- the SHARP trial comparing sorafenib versus placebo $^{3}$ - assessment of the secondary endpoint, time to progression, was already conducted by centralized radiologic review assessment using some of the criteria that ultimately were the embryo for the current AASLD-JNCI and mRECIST criteria summarized herein. The proposed mRECIST assessment is expected to provide a reliable method for assessing tumor response in HCC clinical trials. Of course, these new criteria need now to pass the same examination as other methods used before. First, at one time point pathologic correlation with tumor measurements will be required. Second, the effect of specific antiangiogenic agents changing the tumor inflow of blood might also have impact in the response assessment. Finally, regulatory agencies have to be persuaded that the mRECIST based on the AASLD-JNCI guidelines is the needed step forward to align the response rates induced by molecular drugs and hard endpoints such as survival. Assessment of response with the mRECIST criteria is necessary to understand the importance in the change of response assessment with novel drugs currently tested in phase II and phase III studies. Hopefully, these criteria will be also adopted in the upcoming updated EASL and AASLD guidelines for HCC management.

\section{ACKNOWLEDGMENTS}

Josep M. Llovet has grants from National Institute of Health-NIDDK 1R01DK076986-01, National Institute of Health (Spain) grant I + D Program (SAF2007-61898), and the Samuel Waxman Cancer Research Foundation.

\section{ABBREVIATIONS}

$\begin{array}{ll}\text { AASLD } & \begin{array}{l}\text { American Association for the Study } \\ \text { of Liver Diseases } \\ \text { computed tomography }\end{array} \\ \text { CT } & \begin{array}{l}\text { European Association for the Study } \\ \text { of the Liver } \\ \text { EASL }\end{array} \\ \text { HCDatocellular carcinoma } \\ \text { mRECIST } & \begin{array}{l}\text { modified RECIST } \\ \text { magnetic resonance imaging }\end{array} \\ \text { MRI } & \begin{array}{l}\text { Response Evaluation Criteria in Solid } \\ \text { Tumors }\end{array} \\ \text { RECIST } & \text { World Health Organization }\end{array}$




\section{REFERENCES}

1. Miller AB, Hoogstraten B, Staquet M, Winkler A. Reporting results of cancer treatment. Cancer 1981;47(1): 207-214

2. Therasse P, Arbuck SG, Eisenhauer EA, et al. New guidelines to evaluate the response to treatment in solid tumors. European Organization for Research and Treatment of Cancer, National Cancer Institute of the United States, National Cancer Institute of Canada. J Natl Cancer Inst 2000;92(3):205-216

3. Llovet JM, Ricci S, Mazzaferro V, et al; SHARP Investigators Study Group. Sorafenib in advanced hepatocellular carcinoma. N Engl J Med 2008;359(4):378-390

4. Forner A, Ayuso C, Varela M, et al. Evaluation of tumor response after locoregional therapies in hepatocellular carcinoma: are response evaluation criteria in solid tumors reliable? Cancer 2009;115(3):616-623

5. Bruix J, Sherman M, Llovet JM, et al; EASL Panel of Experts on HCCEuropean Association for the Study of the Liver. Clinical management of hepatocellular carcinoma. Conclusions of the Barcelona-2000 EASL conference. J Hepatol 2001;35(3):421-430

6. Bruix J, Sherman M; Practice Guidelines Committee, American Association for the Study of Liver Diseases.
Management of hepatocellular carcinoma. Hepatology 2005; 42(5):1208-1236

7. Llovet JM, Di Bisceglie AM, Bruix J, et al; Panel of Experts in HCC-Design Clinical Trials. Design and endpoints of clinical trials in hepatocellular carcinoma. J Natl Cancer Inst 2008;100(10):698-711

8. D'Amico G, Garcia-Tsao G, Pagliaro L. Natural history and prognostic indicators of survival in cirrhosis: a systematic review of 118 studies. J Hepatol 2006;44(1):217-231

9. Llovet JM, Burroughs A, Bruix J. Hepatocellular carcinoma. Lancet 2003;362(9399):1907-1917

10. Llovet JM, Bruix J. Systematic review of randomized trials for unresectable hepatocellular carcinoma: Chemoembolization improves survival. Hepatology 2003;37(2):429-442

11. Eisenhauer EA, Therasse P, Bogaerts J, et al. New response evaluation criteria in solid tumours: revised RECIST guideline (version 1.1). Eur J Cancer 2009;45(2):228-247

12. Bogaerts J, Ford R, Sargent D, et al; RECIST Working Party. Individual patient data analysis to assess modifications to the RECIST criteria. Eur J Cancer 2009;45(2):248-260

13. Burrel M, Llovet JM, Ayuso C, et al; Barcelona Clínic Liver Cancer Group. MRI angiography is superior to helical CT for detection of $\mathrm{HCC}$ prior to liver transplantation: an explant correlation. Hepatology 2003;38(4):1034-1042 